\title{
Echocardiographic-anatomical correlations in aorto-left ventricular tunnel
}

Andrew C Cook, Nuala L K Fagg, Siew Yen Ho, Alison M M Groves, Gurleen K Sharland, Robert H Anderson, Lindsey D Allan

\begin{abstract}
Objective-To investigate the echocardiographic, morphological, and histological appearances of aorto-left ventricular tunnel observed in four fetal hearts and compare the findings with those reported in older patients with the malformation. Background-Previous studies have concentrated on clinical features of the malformation from birth to adult life and have speculated on either its embryological formation or its acquisition during late intrauterine life. The presentation of a large series of cases in fetal life is a unique opportunity to study the malformation at an early stage in its natural course.

Methods-A retrospective study was per-
\end{abstract} formed of four cases of aorto-left ventricular tunnel discovered among 872 cases of congenital abnormalities diagnosed at a tertiary centre for fetal echocardiography. Detailed echocardiographic and anatomical observations were made of the malformation as identified during fetal life. The precise anatomical arrangement was determined and compared with previous descriptions found in journals published in English.

Results-In fetal life, as after birth, the malformation is characterised by enlargement and hypertrophy of the left ventricle, enlargement of the aortic root, and free regurgitation at the level of the aortic valve. Anatomical abnormalities are found at the aortic ventriculoarterial and sinutubular junctions as well as in the intervening aortic wall. These are unrelated to necrosis, ischaemia, or the presence of mucopolysaccharides.

Conclusions-The lesion is a developmental abnormality that should be reliably diagnosed by fetal echocardiography combined with colour flow Doppler echocardiography during the midtrimester. The exact anatomical relations clarified by this study are pertinent to diagnosis and subsequent surgical correction.

(Br Heart f 1995;74:443-448)

Keywords: aorto-left ventricular tunnel; echocardiographic-anatomical correlates; developmental malformation; fetal heart.

An aorto-left ventricular tunnel is an extremely rare lesion. This malformation was first recognised in $1961^{1}$ and the anatomic features were subsequently emphasised by Levy $e t a l .^{2}$ Since then approximately 71 cases have been reported in the English literature. The clinical profile ${ }^{3-5}$ and echocardiographic features ${ }^{6-12}$ are well described, but the precise anatomy remains difficult to understand.

We have now encountered four cases of aorto-left ventricular tunnel detected by echocardiography during intrauterine life. Careful analysis of this material has permitted us to clarify the morphological arrangement and histology of the lesion, its relation to adjacent cardiac structures, and suggest criteria to permit its future echocardiographic diagnosis even during fetal life.

\section{Material and methods}

ECHOCARDIOGRAPHY

Four fetuses $(0.5 \%)$ from a series of 872 cases referred to our specialist centre between 1980 and 1992 had an aorto-left ventricular tunnel. Detailed examination was performed during fetal life using an Advanced Technical Laboratories Ultramark 3 mechanical sector scanner in one case and an Advanced Technical Laboratories Ultramark 4 and Hewlett Packard 77020 phased array imaging systems with $5 \mathrm{MHz}$ or $3.5 \mathrm{MHz}$ transducers in three. Doppler evaluation was performed in three cases and colour flow mapping in two.

\section{ANATOMICAL AND HISTOLOGICAL ANALYSIS}

Complete postmortem examination was carried out in each case. Three fetuses died during gestation, while the fourth patient died during the neonatal period. All external and internal abnormalities were documented, the heart was fixed by inflation with formalin, and dissection subsequently carried out following a standard procedure. ${ }^{13}$

A single representative case (case 2) was selected for histological examination after careful documentation. The heart was imbedded in paraffin wax and sectioned serially at a thickness of $10 \mu \mathrm{m}$. All sections were retained according to a standard procedure ${ }^{14}$ and initially each 25 th section was mounted and stained with the trichome technique. Further sections were mounted and stained as required. Staining with alcian blue was performed to determine the presence of acid mucopolysaccharides. Comparisons were made with serial sections of the aortic root of a normal fetal heart of the same gestational age. 


\section{Results}

ECHOCARDIOGRAPHIC FEATURES

The mean (range) gestational age at referral to our centre was 23 (18-33) weeks. An initial diagnosis of aneurysm of the sinus of Valsalva with aortic stenosis and incompetence was made in the first patient at 33 weeks. Failure to respond to medical treatment after birth resulted in surgical intervention at 4 months. A tunnel found arising above the right coronary aortic sinus and leading into the left ventricle was closed. The patient died 2 weeks after operation despite full medical support. The findings in the second fetus (seen at 19 weeks) simulated the anatomical configuration of Fallot's tetralogy with aortic regurgitation. In the third fetus (seen at 18 weeks) the leaflets of the aortic valve were thickened, there was dilatation of its orifice, and the aorta
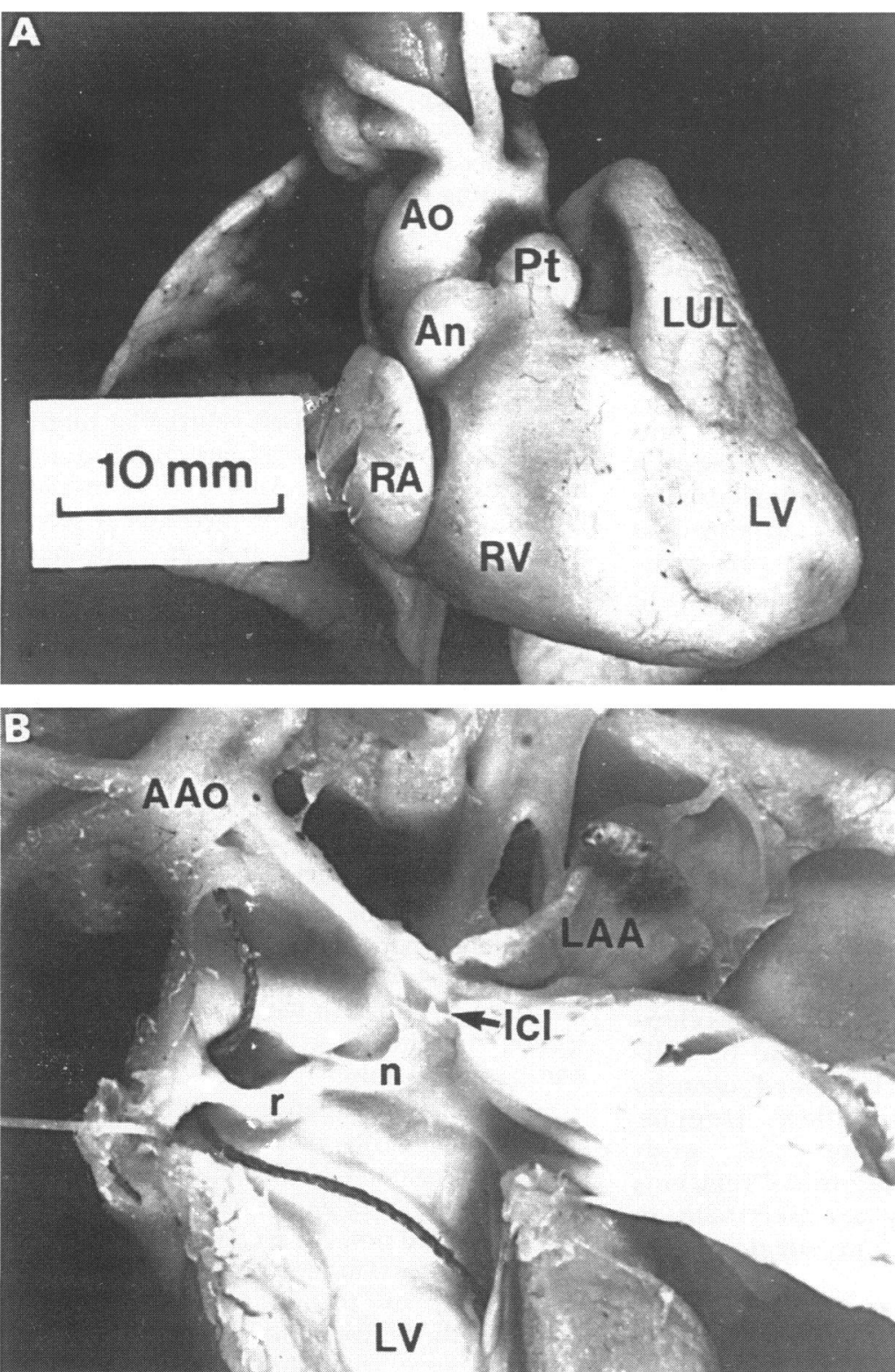

Figure 1 (A) Example of aorto-left ventricular tunnel from a 19 week fetus showing moderate hypoplasia of the left lower lobe and aneurysmal dilatation of the aortic root (An). (B) Left ventricular outflow tract of the same fetus has been opened to demonstrate orifices of the tunnel proximally within the intercoronary interleaflet triangle and distally above the sinutubular junction to show its course. The ventricular opening is relatively small. Ao, aorta; $A A o$, ascending aorta; An, aneurysm; $L A A$, left atrial appendage; lcl, left coronary leaflet; LUI, left upper lobe; $n$, non coronary leaflet; $P t$, pulmonary trunk, $R A$, right atrium; $r$, right coronary leaflet; $R V$, right ventricle; $L V$, left ventricle. was aneurysmically dilated beyond the valve. The overall diagnosis was of profound aortic stenosis and incompetence. The fourth fetus (seen at 22 weeks) was again referred as a case of tetralogy of Fallot. In this fetus, however, the apparently deficient area of the ventricular septum, thought initially to represent a ventricular septal defect with aortic override, was shown to be a tunnel through which turbulent flow passed from the left ventricle to the aorta. The left ventricle in the four fetuses was not echogenic but dilated and thick walled with a decreased shortening fraction.

Parents of the last three fetuses elected to interrupt the pregnancy and the heart was subsequently obtained for study and comparison with the echocardiographic scans.

\section{ANATOMICAL FINDINGS}

Two fetuses were male. There were no external dysmorphic features and the only extracardiac anomaly found was a Meckel's diverticulum (case 2).

All hearts showed similar external appearances. There was pronounced enlargement of the left ventricle, which was approximately two to four times the size of the right ventricle and most notable in the neonatal heart. The aorta was enlarged in each fetal specimen (cases 2-4) with a diameter of $160-200 \%$ greater than mean normal values for the respective gestational ages. In general, the ascending aorta was uniformly dilated at its root but, in one fetal specimen (now 19 weeks gestation by foot length, case 3 ) there was an additional and distinctive aneurysmal bulge at the site of the right coronary aortic sinus (fig 1(A)). The aortic end of the tunnel in the neonatal specimen had been closed during operation by resection of the aneurysmal dilatation over the right coronary sinus and suturing of the aortic wall to the fibrous bar supporting the right coronary aortic leaflet. There was also noticeable bulging of the ventricular septum into the posterior portion of the right ventricular outflow tract in this specimen.

All hearts showed normal connections of the cardiac segments and usual venoatrial connections. The origin of the tunnel in each case was at the site of the interleaflet triangle between the attachments of the valvar leaflets in the left and right coronary aortic sinuses. It then extended to varying degrees inferiorly beneath the right coronary aortic leaflet, which arose from a fibrous bar spanning the aortic root. The inferior aspect of the opening showed varying degrees of dilatation (figs 1(B) and 2). The tunnel itself was located in the fibro-fatty tissue plane separating the aortic and pulmonary trunks, with its right side bulging into the posterior portion of the right ventricular infundibulum. As the tunnel spiralled superiorly and to the right, it bypassed the hinge point of the right coronary leaflet of the aortic valve arising from the fibrous bar within the aortic root. In each case the tunnel led into the ascending aorta above the sinutubular junction of the right coronary sinus. The mid-point of the bar supporting the aortic 


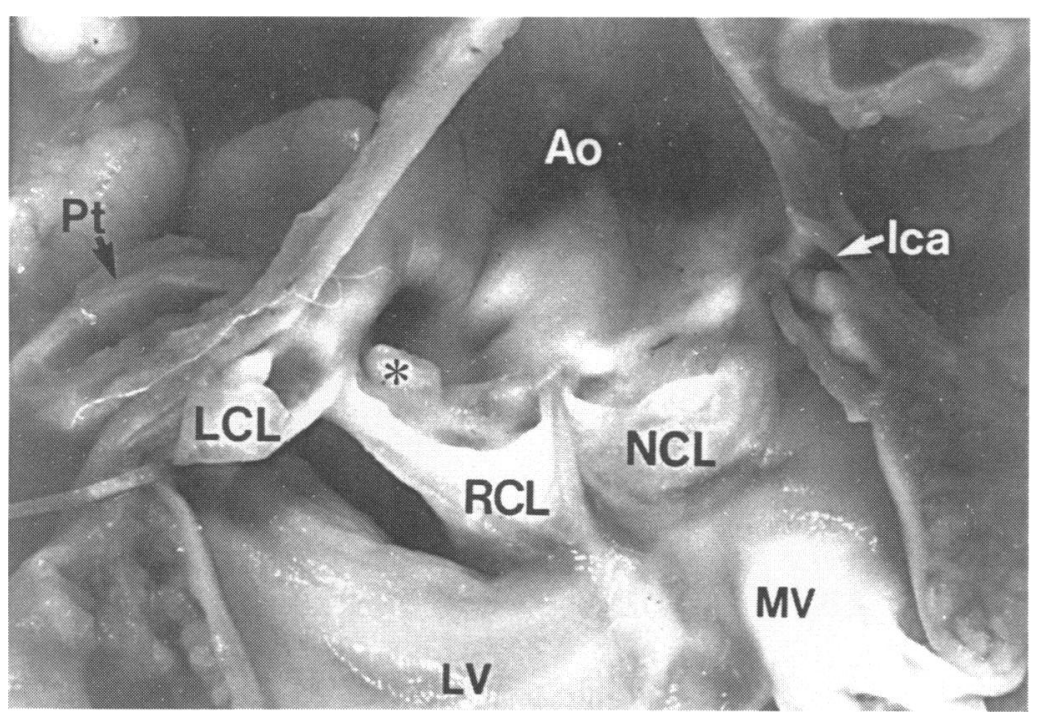

Figure 2 Inferior aspect (as in fig 1(B)) of the orifices of the tunnel in a 22 week fetus, showing the consistent position of the openings of the tunnel. There is valve-like mucoid tissue present on the aortic aspect of the fibrous ridge ( $\left.{ }^{\star}\right)$. lca, left coronary artery; $M V$, mitral valve; NCL, non-coronary sinus; RCL, right coronary sinus; other abbreviations as given in fig 1. along this junction and rises to the apex of the interleaflet triangle (fig 3 ). In case 2 reported here the aortic wall beneath the right coronary leaflet was deficient and as a consequence the ventriculoarterial junction was anomalous. The right coronary leaflet itself was fixed on its upper surface to the fibrous bar spanning the aortic root (fig 4(B)). The leaflet and its supporting fibrous bar, therefore, formed the roof of the entrance of the tunnel into the left ventricle, the floor being formed by left ventricular myocardium (fig $4 \mathrm{~A}$ and $\mathrm{B}$ ).

The intercoronary aortic interleaflet triangle in the normal fetal heart is bordered anteriorly by the wall of the pulmonary trunk, the two structures being separated from one another by a fibro-fatty tissue plane (fig 3 ). In the abnormal heart the tunnel passed from the left ventricle into this fibro-fatty tissue plane. It did not pass through the wall of the right ventricular infundibulum, nor through the outlet septum, but merely displaced the wall of the right ventricular outflow tract (fig 4(A)). Thus, the inferior border of the tunnel was formed by the septal myocardium. The roof of the tunnel, however, was more arterial in composition (fig 4(B)). Its epicardial aspect consisted of loose vascularised collagen and sparse myofibres-similar structures being seen in the epicardial region of the normal right ventricle adjacent to the intercoronary aortic commissure. Internally, the roof was

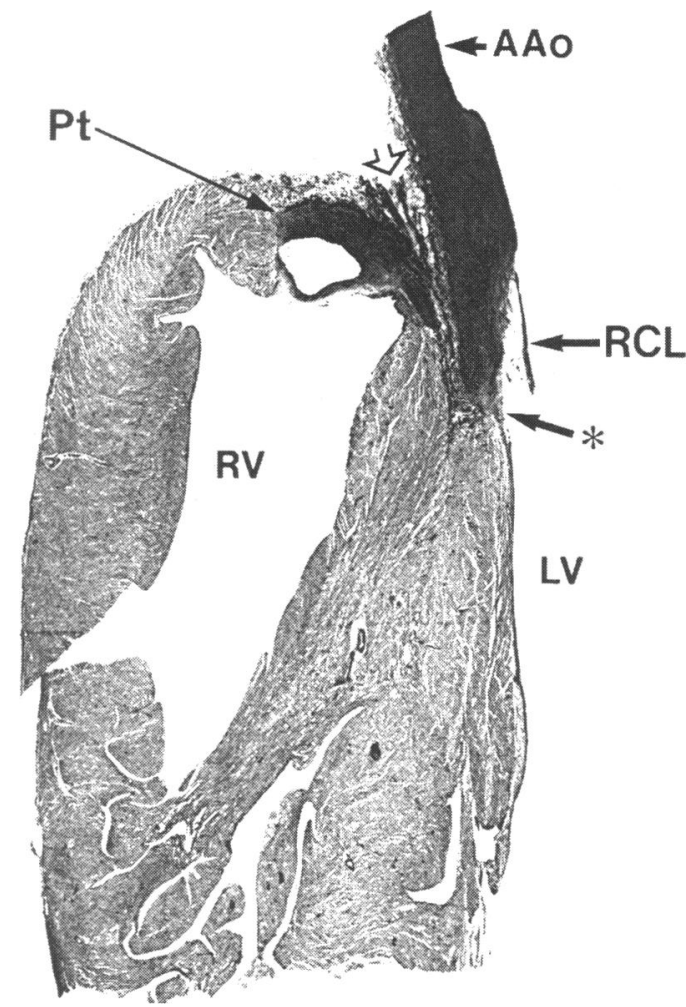

Figure 3 Section from a normal fetal heart taken across the tissue plane (open arrow) between the aorta and pulmonary trunk through which the tunnel passes, showing $a$ well defined collagenous junction between the base of the aortic wall and ventricular myocardium in this region $(*)$. This junction was absent in the fetal specimens of aorto-left ventricular tunnel. Furthermore, the section shows that the mid-point of the interleaflet triangle abutts onto the mid-point of the interleaflet triangle abutts onto the
pulmonary trunk and the posterior portion of the right ventricular infundibulum; a relation conserved in aorto-left ventricular tunnel. of the fibrous triangle, the aortic wall is joined to the ventricular myocardium by a layer of collagen (the anatomical ventriculoarterial junction). The right coronary leaflet attaches

leaflet extended in one case to a point level case the orifice of the right coronary fibrous bar supporting the aortic valvar leaflet adjacent leaflets of the aortic valves, and ening of the valvar leaflets while, in the fourth fibrous bar that separated the tunnel from the ight coronary sinus (fig 2) anterocephalad to the area of the membra(see later), the left bundle branch could be membranous septum and took a normal

HISTOLOGICAL FINDINGS

The fibrous triangle separating the coronary leaflets of the aortic valve in the normal heart has an elastic media and is of similar thickness 


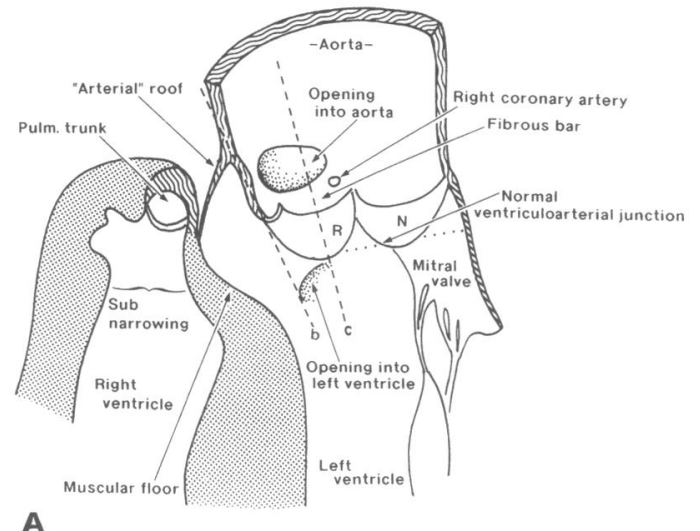

A

Figure 4 (A) Schematic representation of aorto-left ventricular tunnel, taken in the same plane as in fig 3, indicating the position of the histological sections in (B) and (C). (B) At the left ventricular opening of the tunnel (closed arrow) the right coronary leaflet is fixed only on its upper surface to the fibrous bar $(F)$ separating the tunnel $(T)$ from the aortic valve. The ventriculoarterial junction beneath is abnormally formed. The floor of the tunnel consists of infundibular myocardium, while the roof is arterial in structure. (C) This arrangement persists until reattachment of the right coronary leaflet to the left ventricular myocardium (closed arrow) from which point the entire tunnel is arterial in structure. $R$ and $N$ as in fig 1. Open arrow, opening of tunnel into aorta; pulm, pulmonary; other abbreviations as given in fig 1. (Elastic van Geison's stain).

formed by a thick layer of fibrous tissue formed by intermingled collagen and elastic fibres. This configuration, of an arterial roof and a muscular floor, persisted along the tunnel until it reached the point of reattachment of the right coronary leaflet of the aortic valve to the left ventricular myocardium. From this region the tunnel was completely arterial in structure (fig $4(\mathrm{C})$ ). At the junction of the tunnel with the ascending aortic wall the elastic fibres were continuous, well laminated and showed no signs of disorganisation. The right coronary artery joined the wall of the tunnel in this region. Mucopolysaccharides were not detected at any point within the tunnel.

\section{Discussion}

Aorto-left ventricular tunnel is a rare anomaly. In our review of the English literature, we have found only 71 cases reported in the 30 years since the original description by Edwards ${ }^{1}$ and its elaboration by Levy et al. ${ }^{2}$ The four cases reported here form, to the best of our knowledge, the largest detailed pathological study of this entity. As well as including, as far as we know, the only cases reported during fetal life, our experience has also enabled us to elucidate the anatomical features since before our study we had not totally understood the existing anatomical descriptions.

\section{ANATOMICAL FEATURES}

Existing descriptions and diagrams of the anatomical relation of the tunnel to the aortic valve and its supporting structures are, at best, ambiguous. Indeed, descriptions of the aortic and left ventricular origins are given in only six of 43 reports. Furthermore, such descriptions are usually confused by reference to an aortic "annulus" or "ring".

The leaflets of the aortic valve, however, do not join the aortic wall in the form of a circlet, but have semilunar attachments. ${ }^{15}$ Such an arrangement gives rise to spaces on the ven- tricular aspects of the leaflets, which can be termed the interleaflet triangles. The base of these triangles is formed by the anatomical ventriculoarterial junction and the apex by the peripheral attachment of the commissures at the level of the sinutubular junction. The triangles themselves are filled by an elastic wall of comparable thickness to the walls of the adjacent aortic sinuses.

In the tunnel studied histologically (case 2) the major disorder was found within the interleaflet triangle between the left and right coronary sinuses. Review of the literature shows this region to be described as the site of the ventricular opening in a further 11 (of 29) cases with tunnels. In the remaining 18 cases the origin is stated to be immediately below the attachments of the aortic valve, although its exact site is not specified. In our case the tunnel was an expansion of the interleaflet triangle that burrowed into the tissue plane between the front of the aorta and the back of the subpulmonary infundibulum. This expansion had the effect of separating the attachment of the right coronary leaflet of the aortic valve from its ventricular support, the leaflet being attached to a dense fibrous bar that spanned the root of the right aortic sinus. The walls of the tunnel were made of fibroelastic tissue, but the base was the crest of the muscular septum. The tunnel itself, therefore, did not extend through the ventricular septum, as has previously been reported. ${ }^{24}$ Instead, it occupied the tissue plane between the subpulmonary infundibulum and the right aortic sinus, deforming the sinus by its position, but lying on the ventricular musculature. It is this expansion of the tunnel within the tissue plane which, by impinging on the infundibulum, produces subpulmonary narrowing, a feature reported in 31 of 71 reviewed cases. The normal anatomical relation of the aorta, pulmonary trunk, and subpulmonary infundibulum, however, are conserved in the presence of a tunnel. 


\section{SURGICAL IMPLICATIONS}

Results of our study suggest that the difficulty in performing surgical closure without distortion of the aortic valve, a feature emphasised in several previous descriptions, ${ }^{5616}$ is caused partly by the overall dilatation of the aortic root and the unsupported nature of the right aortic valvar leaflet. Any attempt to suture the bar of tissue supporting this leaflet directly to the wall of the tunnel is likely to result in distortion, as the ridge supports the hinge point of the leaflet in its anatomically appropriate position. Thus, closure of the aortic origin of the tunnel with a patch would seem more anatomically correct. Care must be taken, nonetheless, to avoid the origin of the right coronary artery as this commonly arises from the lateral wall of the tunnel (38\% of reviewed cases, and three of the four reported here). Closure of the left ventricular end of the tunnel within the interleaflet triangle has also been described. ${ }^{16} 17$ Sutures for such closure can be placed within the muscle separating the tunnel from the membranous septum. They should not generate any possibility for creating heart block unless placed very deeply.

Many authors have suggested that early operation is important to prevent distortion of the aortic valve and the root. ${ }^{461624}$ Although significant abnormalities of the aortic valve were not found in the fetal specimens, profound dilatation of the aortic root associated with aortic regurgitation was already present in early gestation. Moreover, accessory valvelike tissue, similar to that seen in two previous cases $^{918}$ was present on the aortic aspect of the fibrous bar in our case of 22 weeks' gestation. Thus it seems that abnormalities of the aortic root are part of the malformation itself and may not be avoided by early surgical closure. It is clear that the method of surgical closure should be tailored to suit the peculiarities of a given tunnel. Excellent medium-term results have been obtained in one centre using this methodology with only one cardiac related death in 13 infants. ${ }^{19}$

\section{ECHOCARDIOGRAPHIC DIAGNOSIS}

The diagnosis of aorto-left ventricular tunnel has frequently been made in postnatal life by cross sectional echocardiography and colour flow imaging. ${ }^{7810-12}$ Our overall experience in fetal echocardiography ${ }^{20}{ }^{21}$ suggests that diagnosis should be feasible during intrauterine life. Yet the correct diagnosis was made in only one of our four cases. The presently described experience should facilitate much more accurate diagnosis in the future. All of our cases showed ultrasonic evidence of free aortic regurgitation at the level of valvar leaflets. Congenital incompetence across the orifice of the aortic valve is rare. ${ }^{22}$ It may occur in postnatal life in several conditions, for instance in aortic stenosis, Marfan's syndrome, as a complication of bacterial endocarditis, or in association with some ventricular septal defects. To our knowledge, none of these combinations has been reported in the fetus. The finding should, therefore, suggest the possibility of a tunnel, it was significant nonetheless that echocardiography in one of our cases was interpreted as showing the aortic valve overriding a ventricular septal defect. This feature, coupled with aortic dilatation and narrowing of the subpulmonary infundibulum, led to the erroneous diagnosis of tetralogy of Fallot with aortic regurgitation. The most recent case had also been referred with the working diagnosis of tetralogy of Fallot. Our morphological experience with the previous case then allowed the correct diagnosis to be made in fetal life.

An aorto-left ventricular tunnel should therefore, be suspected in any fetus with pronounced regurgitation of the aortic valve, noticeable enlargement of the aortic root, and conspicuous hypertrophy of the left ventricle. We are unable to explain why this combination should be so relatively common in our fetal experience, yet be seen so rarely in other postnatal centres. ${ }^{5}$

\section{AETIOLOGY}

Our finding of aorto-left ventricular tunnel in the early mid-trimester confirms the suspicions of Levy $e t a l^{2}$ that the malformation is of developmental origin rather than representing an acquired disease of postnatal life. The lesion could, nonetheless, be acquired during fetal life subsequent to the completion of cardiac septation. Our histological findings confirm the structure of the tunnel during fetal life to be similar to that reported by Levy et $a l .{ }^{2}$ We found no evidence of ischaemia, necrosis, or rupture in our case examined by serial sectioning. If such processes are involved in the origin of the malformation, as suggested by some authors, ${ }^{3}{ }^{19}{ }^{23-25}$ then they must occur before 22 weeks' gestation and subsequently disappear, an evolution which seems unlikely. Furthermore, we were unable to find acid mucopolysaccharides in the extracellular matrix of the wall of the tunnel. Absence of this feature suggests that an aetiological link with Marfan's syndrome, as suggested by Cooley et $a l l^{3}$ is improbable. It is possible, nonetheless, that part of the tunnel is formed as a result of anomalous formation of the right coronary artery. The aortic end of the tunnel is arterial in structure and intimately related to, and often incorporates, the origin of the right coronary artery. Indeed, the passage of the distal end of the tunnel through the aortic wall is distinctly reminiscent of an intramural coronary artery. ${ }^{26}$ Formation of the left ventricular opening of the tunnel, its consistent position, and the associated disturbance of the ventriculoarterial junction are more difficult to explain. Evidence concerning their genesis will require examination of very early fetal specimens.

We are grateful to Lucienne Kilpatrick, Department of Paediatrics, National Heart and Lung Institute, London, for her expertise in preparing the serial sections and to Sarah-Jan Smith, Department of Anatomy, UMDS Guy's Hospital, London, for her skilful production of the photomicrographs. ACC, SYH, RHA, and LDA are supported by the British Heart Foundation; RHA is also supported by the Joseph Levy Foundation. 
1 Edwards JE. Atlas of acquired disease of the heart and great vessels. Vol III. Philadelphia: WB Saunders, 1961:1142.

2 Levy MJ, Lellehei CW, Anderson RC, Amplatz K, Edwards JE. Aortico-left ventricular tunnel. Circulation 1963;27:841-53.

3 Cooley RN, Harris LC, Rodin AE. Abnormal communication between the aorta and left ventricle-aortico-left ventricular tunnel. Circulation 1965;31:564-71.

4 Somerville J, English T, Ross DN. Aorto-left ventricular tunnel. Clinical features and surgical management. $\mathrm{Br}$ Heart $\mathcal{F}$ 1974;36:321-8.

5 Okoroma EO, Perry LW, Scott LP, McClenathan JE. Aortico left ventricular tunnel. $\mathcal{F}$ Thorac Cardiovasc Surg Aortico left ventric

6 Sreeram N, Franks R, Walsh K. Aortic-ventricular tunnel in a neonate: diagnosis and management based on cross sectional and colour Doppler ultrasonography. Br Heart $\mathcal{f}$

7 Turley K, Silverman NH, Teital D, Mavroudis C, Snider R, Rudolph A. Repair of aortico-left ventricular tunnel in the neonate, surgical, anatomic and echocardiographic considerations. Circulation 1982;65:1015-20.

8 Perry JC, Nanda NC, Hicks DG, Harris JP. Two-dimensional echocardiographic identification of aortico-left ventricular tunnel. Am f Cardiol 1983;52:913-4.

9 Bash SE, Huhta JC, Nihill MR, Vargo TA, Hallman GL. Aortico-left ventricular tunnel with ventricular septal defect two-dimensional/Doppler echocardiographic diagnosis. $₹$ Am Coll Cardiol 1985;5:757-60.

10 Humes RA, Hagler DJ, Julsrud PR, Levy JM, Feldt RH, Schaff HV. Aortico-left ventricular tunnel diagnosis based on two-dimensional echocardiography colour flow based on two-dimensional echocardiography colour flow Doppler imaging and magne

11 Fripp RR, Werner JC, Whitman V, Nordenberg A, Waldhausen JA. Pulsed Doppler and two-dimensional echocardiographic findings in aortico-left ventricular tunnel. $₹$ Am Coll Cardiol 1984;4:1012-4.

12 Akalin H, Erol, Oral D, et al. Aortico-left ventricular tunnel: successful diagnostic and surgical approach to the oldest patient in the literature. 7 Thorac Cardiovasc Surg 1989; 97(5):804-5.

13 Devine WA, Debich DE, Anderson RH. Dissection of congenitally malformed hearts with comments on the value of sequential segmental analysis. Pediatr Patho

14 Smith A, Ho SY, Anderson RH. Histological study of the cardiac conducting system as a routine procedure. Med Lab Sci 1977;34:223-9.

15 Anderson RH, Devine WA, Ho SY, Smith A, McKay R The myth of the aortic annulus: the anatomy of the
subaortic outflow tract. Ann Thorac Surg 1991;52:640-6.

16 Hovaguimian $\mathrm{H}$, Cobanoglu A, Starr A. Aortico-left ventricular tunnel-a clinical review and new surgical classification. Ann Thorac Surg 1988;45:106-12.

17 Saylam A, Ozme S, Aslamaci S, Olga R, Aytac A. Aorticoleft ventricular tunnel. Thorac Cardiovasc Surg 1981;29: 259-61.

18 Bove KE, Schwartz DC. Aortico-left ventricular tunnel. A new concept. Am $\mathcal{f}$ Cardiol 1967;19:696-709.

19 Horvath P, Balaji S, Skovranek S, Hucin B, De Leval MR Stark J. Surgical treatment of aortico-left ventricular tunnel. Eur F Cardiothorac Surg 1991;5:113-6.

20 Allan LD, Tynan MJ, Campbell S, Wilkinson J, Anderson RH. Echocardiographic and anatomical correlates in th fetus. Br Heart f 1980;44:444-51.

21 Sharland GK, Allan LD. Screening for Congenital Hear Disease prenatally. Results of $2 \frac{1}{2}$ year study in the South East Thames Region. Br f Obstet Gynaecol 1992;99: $220-5$

22 Levine SA, Harvey PW. Congenital aortic insufficiency. In: Clinical auscultation of the heart. 2nd ed. Philadelphia: In: Clinical auscultation of

23 Spooner EW, Dunn JM, Behrendt DM. Aortico-left ventricular tunnel and sinus of Valsalva aneurysm. $₹$ Thorac tricular tunnel and sinus of Valsalva

24 Roberts WC, Morrow AG. Aortico-left ventricular tunnel $A$ cause of massive aortic regurgitation and of intracardiac aneurysm. Am $\mathcal{Y}$ Med 1965;39:662-7.

25 Gardina ACV, Levin AR, Engle MA. Aortico-left ventricular tunnel with natal cardiac failure. South Med $\mathcal{F} 1977$ 70:1351-54

26 Gittenberger-de Groot AC, Sauer U, Quaegebeur J. Aortic intramural coronary artery in three hearts with transposition of the great arteries. F Thorac Cardiovasc Surg 1986; 91:566-71. 